\title{
Ultra-High Energy Resolution EELS
}

\author{
T.C. Lovejoy ${ }^{1}$, N.J. Bacon ${ }^{1}$, A.L. Bleloch ${ }^{1}$, N. Dellby ${ }^{1}$, M.V. Hoffman ${ }^{1}$, and O.L. Krivanek ${ }^{1,2}$ \\ 1. Nion R\&D, 11511 NE 118th St, Kirkland, WA, USA \\ 2. Department of Physics, Arizona State University, Tempe, AZ, USA
}

The High Energy Resolution Monochromated EELS-STEM (HERMES) system developed by Nion combines a ground-potential monochromator, a bright cold-field-emission (CFE) gun, an advanced aberration corrector, and a new EEL spectrometer. The system is designed to allow < $10 \mathrm{meV}$ energy resolution EELS while maintaining sub-nm spatial resolution at the sample. It is greatly extending the capabilities of vibrational spectroscopy in the electron microscope, which was introduced 3 years ago [1] and has already achieved:

- sub-nm spatial resolution [2]

- damage-free identification of different bonds including hydrogen ones in a guanine sample [3]

- probing atomic vibrations at surfaces and edges of nano-objects, with nm spatial resolution [4].

The HERMES system has recently attained 7.1 meV FWHM (full width at half-maximum) of the zero loss peak (ZLP) at $60 \mathrm{kV}$ - see Figure 1. However, the theoretical diffraction-limited energy resolution of the system is around $1 \mathrm{meV}$. Taking $5 \mathrm{meV}$ as a practical near-term goal, this presentation will describe our efforts to bridge the gap between theory and practice via:

Optimizing the size of the slit-level crossover, $D_{\text {slit, }}$ which together with the energy dispersion at the slit, Disp, determines the ultimate energy width of the electrons at the sample by $D_{\text {slit }} /$ Disp. In exact analogy with the way the sample-level crossover (i.e. the demagnified image of the electron source, also known as "the probe") is formed, $D_{\text {slit }}$ is determined by the diffraction-limited size of the source image, $D_{\text {diff }}$, given by the incident angles (chosen so that aberrations do not affect the probe formation), and broadened by finite source size according to

$$
\left(D_{\text {slit }}\right)^{2}=\left(D_{\text {diff }}\right)^{2}\left(1+\left(I / I_{c}\right)^{2}\right)
$$

where $I$ is the current incident on the slit, and $I_{c}$ is the coherent current of the electron source [5]. Using the slit to select $5 \mathrm{meV}$ out of the intrinsic $300 \mathrm{meV}$ CFE energy width reduces the current by $\sim 60 \mathrm{x}$ (or more). The minimum useful post-slit beam current for spectroscopy is about $5 \mathrm{pA}$, so it is necessary to use a pre-slit beam current $I>300 \mathrm{pA}$. For the CFE gun, $I_{c} \sim 200 \mathrm{pA}$, which means that the source size at the slit has a significant influence on the attainable energy resolution.

Also in analogy with probe formation, the effect of finite source size can be reduced by increasing the angles (decreasing $D_{\text {diff }}$ ) - i.e. by correcting the aberrations of the image of the source at the slit, and increasing the range of admitted angles correspondingly. The Nion monochromator includes sextupoles and octupoles before the slit for this purpose, and using an auto-tuning algorithm adapted from probe corrector tuning, it is straightforward to correct $2^{\text {nd }}$ order aberrations at the slit. $3^{\text {rd }}$ order correction is also available, though less important in a monochromator using magnetic prisms, which have large second-order aberrations.

Minimizing the instabilities in the total system, which can broaden the slit-level crossover, the probe at the sample, and the crossover on the final EELS detector. The monochromator system design includes 
stabilization schemes [6] that, together with a stable microscope environment and ultra-stable power supplies, effectively reduce long-term drift. Pre-sample instabilities can be measured quantitatively using scanned images, which typically show instabilities $<<$ one diffraction limit. Post-sample instabilities can be also be quantified, by fast recording of the position of the ZLP on the EELS detector. Typical EELS instabilities have been much larger than the diffraction limit, which points to the postsample column, especially the pre-EELS detector chamber and the EELS, as the weak link in the stability picture. Our new spectrometer applies the same rigorous design standards as those used in the pre-sample column, such as mechanical rigidity and multiple $\mu$-metal shielding, all the way from the sample to the final detector.

Minimizing the size of the crossover at the final detector on the EELS, which is similar to the crossover at the slit, except that the energy dispersion must be magnified more because the detector pixels are typically 10-20 $\mu \mathrm{m}$ in size. Once the instabilities, both short-term shakes and long-term drift (and tails, see below), are no longer limiting, we can focus on EELS aberration correction and aim for high-angle collection (>50 mrad half-angle) combined with <10meV energy resolution.

The FWHM of the ZLP is a key factor, but practical work relies on further factors including:

- The Full-Width at 1/10,000 maximum. Minimizing this requires careful management of the tails of the ZLP both from the monochromation system, and in the EELS.

- Flexible camera lengths allowing EELS collection angles ranging from micro-radians (at the sample, for high momentum resolution experiments) to $>50 \mathrm{mrad}$.

- Ease of use and appropriate automation so that all the available modes are accessible with only moderate training.

Our progress in each of these areas will be described in the presentation.

In summary, as the EELS energy resolution approaches $5 \mathrm{meV}$, the requirements on the system, from the source to the spectrometer, increase substantially. Optimization in the key areas described above will lead to an era of diffraction-limited EELS in which $5 \mathrm{meV}$ and ultimately 1-2 meV should be possible. The benefits for vibrational spectroscopy promise to be immense, especially for biological samples, in which $<5 \mathrm{meV}$ resolution will allow us to identify many different types of molecules.

References:

[1] OL Krivanek et al, Nature 514 (2014) p. 209.

[2] C Dwyer et al, Phys. Rev. Lett. 117 (2016) p. 256101.

[3] P Rez et al, Nat. Comms. 7 (2016) 10945

[4] MJ Lagos and PE Batson, Nature, accepted (2017)

[4] OL Krivanek et al, in: Scanning Transmission Electron Microscopy, SJ Pennycook and PD Nellist, Eds (Springer, 2010) p. 615.

[6] OL Krivanek et al, Phil. Trans. R. Soc. A367 (2009) p. 3683.

Figure 1. ZLP with $7.1 \mathrm{meV}$ FWHM acquired at $60 \mathrm{kV}$ in vacuum in $3 \mathrm{~ms}$ with $\beta / 2=10 \mathrm{mrad}$, and energy dispersion at the spectrometer detector of $0.5 \mathrm{meV} / \mathrm{channel}$. The current before the

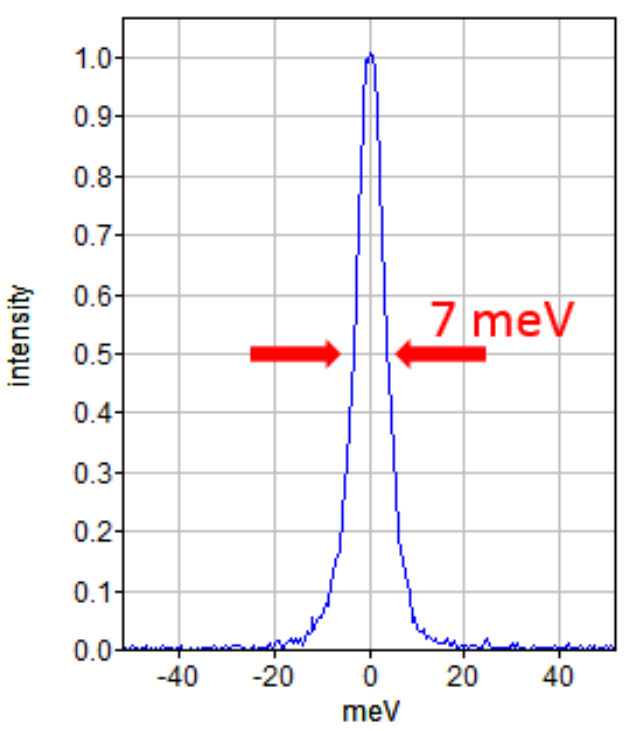
slit was $\sim 300 \mathrm{pA}$, the post-slit beam current $\sim 10 \mathrm{pA}$. 